\title{
Microscopic origins of the ferromagnetic exchange coupling in oxoverdazyl-based $\mathrm{Cu}$ (II) complex
}

\author{
Jean-Baptiste Rota, ${ }^{1}$ Carmen J. Calzado, ${ }^{2}$ Cyrille Train, ${ }^{3}$ and Vincent Robert ${ }^{1, a)}$ \\ ${ }^{1}$ Laboratoire de Chimie, Ecole Normale Supérieure de Lyon, CNRS, Université de Lyon, 46 Allée d'Italie, \\ F-69364 Lyon, France \\ ${ }^{2}$ Departamento de Quimica Fisica, Universidad de Sevilla, c/Prof. Garcia Gonzalez, s/n E-41012 Sevilla, \\ Spain \\ ${ }^{3}$ LNCMI, UPR CNRS 3228, 25 Rue des Martyrs, B.P. 166, 38042 Grenoble Cedex 9, France \\ and Université Joseph Fourier, BP 53, F-38041 Grenoble Cedex 09, France
}

(Received 18 January 2010; accepted 12 March 2010; published online 20 April 2010)

\begin{abstract}
The exchange channels governing the experimentally reported coupling constant $\left(J_{\text {expt }}=6 \mathrm{~cm}^{-1}\right)$ value in the verdazyl-ligand based $\mathrm{Cu}(\mathrm{II})$ complex $\left[\mathrm{Cu}(\mathrm{hfac})_{2}(\mathrm{imvdz})\right]$ are inspected using wave function-based difference dedicated configuration interaction calculations. The interaction between the two spin $1 / 2$ holders is summed up in a unique coupling constant $J$. Nevertheless, by gradually increasing the level of calculation, different mechanisms of interaction are turned on step by step. In the present system, the calculated exchange interaction then appears alternatively ferromagnetic/ antiferromagnetic/ferromagnetic. Our analysis demonstrates the tremendously importance of some specific exchange mechanisms. It is actually shown that both parts of the imvdz ligand simultaneously influence the ferromagnetic behavior which ultimately reaches $J_{\text {calc }}=6.3 \mathrm{~cm}^{-1}$, in very good agreement with the experimental value. In accordance with the alternation of $J$, it is shown that the nature of the magnetic behavior results from competing channels. First, an antiferromagnetic contribution can be essentially attributed to single excitations involving the $\pi$ network localized on the verdazyl part. In contrast, the $\sigma$ ligand-to-metal charge transfer (LMCT) involving the imidazole moiety affords a ferromagnetic contribution. The distinct nature $\sigma / \pi$ of the mechanisms is responsible for the net ferromagnetic behavior. The intuitively innocent part of the verdazyl-based ligands is deeply reconsidered and opens new routes into the rational design of magnetic objects. (C) 2010 American Institute of Physics. [doi:10.1063/1.3378023]
\end{abstract}

\section{INTRODUCTION}

Much interest has been devoted to the synthesis of molecule-based magnetic systems since the flexibility offered by such systems considerably extends this important field of research. ${ }^{1-6}$ Consequently, the interpretation of the underlying mechanisms has become a real challenge since promising properties can be anticipated in molecular magnetic as well as in extended networks. ${ }^{7-12}$ One of the featuring characteristics of such systems is the temperaturedependent magnetic susceptibility that can be modeled phenomenologically by an exchange coupling constant $J$. The sign and amplitude of this parameter summarize the interactions between a limited number of localized spins. For a two center/two electron prototype system, the singlet-triplet energy difference reads $J$ using a Heisenberg-Dirac-Van Vleck spin-only phenomenological Hamiltonian $H=-J \vec{S}_{1} \cdot \vec{S}_{2}$ with $S_{1}=S_{2}=1 / 2$. For magnetically coupled systems, such Hamiltonian is indeed relevant since the spatial parts of the different spin-state wave functions are very similar. Among the abundant class of multicentric architectures involving open-shell metal ions, paramagnetic ligand-based systems have turned out to be promising targets to enhance the exchange interactions. ${ }^{8,13,14}$ The originality of such sys-

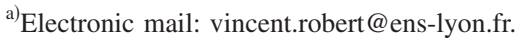

tems stems from the intrinsically molecular character of the magnetic orbitals, in contrast with standard paramagnetic metal ion-based systems. In this respect, thiazyl or verdazyl radical (vdz) containing ligands represent interesting targets toward strongly coupled discrete ${ }^{15-18}$ and extended ${ }^{16-19}$ molecule-based magnetic systems. ${ }^{20-22}$ These systems are particularly stable as compared to other classes of organic radicals that exhibit a strong propensity to dimerization. Several examples based on verdazyl radicals have been reported in literature. ${ }^{23}$ The nature and amplitude of the intramolecular interactions are sensitive to the metal ion and verdazyl radical partner ranging from strong ferromagnetic $\left(\sim 200 \mathrm{~cm}^{-1}\right)$ to antiferromagnetic $\left(\sim-60 \mathrm{~cm}^{-1}\right)$ behaviors. $^{15,24}$

With this goal in mind, Hicks et al. ${ }^{24}$ synthesized and magnetically characterized several copper-verdazyl complexes, tuning the radical design at will, the crystal packing, and the coordination sphere of the metal. Among this class of systems, the $\left[\mathrm{Cu}(\mathrm{hfac})_{2}(\mathrm{imvdz})\right]$ (1) compound (see Fig. 1) has attracted our attention considering its observed ferromagnetic behavior (imvdz=1,5-dimethyl-3-(1-methyl-2imidazolyl)-6-oxoverdayl; hfac $=$ hexafluoroacetylacetonate) .

The exchange interaction between the copper(II) ion and the verdazyl radical has been estimated to $6 \mathrm{~cm}^{-1}$ from temperature-dependent magnetic susceptibility measurements. The splitting of the d-orbitals resulting from a simple 


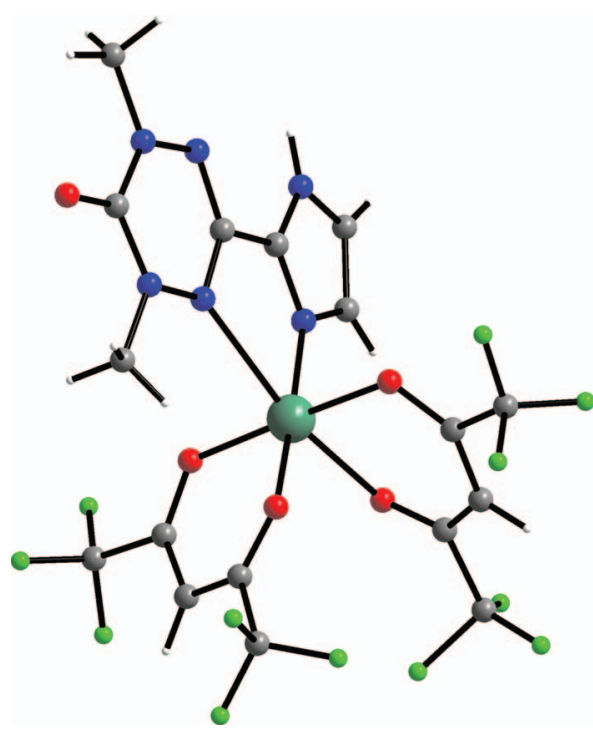

FIG. 1. X-ray structure of the $\left[\mathrm{Cu}(\mathrm{hfac})_{2}(\mathrm{imvdz})\right]$ compound (1) (Ref. 24).

crystal field picture should favor a ferromagnetic behavior. Indeed, their filling leaves a singly occupied d-orbital that is orthogonal to the verdazyl singly occupied molecular orbital (SOMO) ${ }^{24}$ Nevertheless, we felt that complementary theoretical inspections were necessary to unravel the physical mechanisms that subtend this ferromagnetism. Indeed, it has been demonstrated that a crystal field picture may not be appropriate to properly account for the magnetic interactions. Specific contributions that go beyond this picture (i.e., valence-only picture) can contribute up to a few tens of wavenumbers to the $J$ value. ${ }^{25-27}$ The so-called correlation effects involving instantaneous charge and spin changes must be included to reach spectroscopic accuracy. As prime examples, the magnetic behaviors of $\left[\mathrm{Ni}(\mathrm{imvdz})(\mathrm{hfac})_{2}\right]$ and $\left[\mathrm{Mn}(\mathrm{imvdz})(\mathrm{hfac})_{2}\right]$ complexes have been recently elucidated. ${ }^{14}$

Since the ferromagnetism of (1) is weak, conclusions drawn from a naive crystal field picture might thus be misleading. Wave function-based calculations were performed to clarify the origin of the magnetic behavior. A multireference framework was favored over a density functional theorybased strategy since it affords a quantitative analysis of the different underlying mechanisms contributing to the exchange coupling between the $3 \mathrm{~d}$ metal ion and the organic radical ligand. In particular, a valence-bond type description that takes advantage of orthogonal localized orbitals (LOs) can be very enlightening. The importance of the chemical groups and electronic mechanisms [i.e., charge transfer, spin polarization (SP)] were inspected by using two complementary tools. Based on the reported crystal structure, we first selectively turned off some specific channels by freezing the occupations of some identified LOs. Such strategy allows one to discriminate between the different chemical moieties that might contribute to the spin-state hierarchization. Besides, it is also possible to analyze the impact of the different types of configurations contained in the CI space on the $J$ value. By gradually increasing the level of calculation, different mechanisms are turned on step by step, leading to drastic modifications of the calculated exchange coupling constant. This strategy is based on a recently proposed classpartitioned configuration interaction (CI) procedure, ${ }^{27}$ which makes it possible to determine the nature and amplitude of the contributions to the exchange coupling constant arising from different physical mechanisms. Evidently, both frameworks are complementary since some specific bonds can be involved through different mechanisms.

\section{COMPUTATIONAL DETAILS}

The coupling between magnetic moments localized on the metal ion and radical gives rise to two magnetic states, a singlet $(S)$ and triplet $(T)$, separated by $J=E_{S}-E_{T}$. The microscopic origin of the exchange interaction can be investigated by means of quantum theoretical calculations. With this goal in mind, the difference dedicated configuration interaction (DDCI) method has been designed and applied to evaluate vertical energy differences. ${ }^{28}$ First, complete active space self-consistent field (CASSCF) calculations are performed to generate a reference space including the leading electronic configurations in the desired spin multiplicities. To reduce the computational cost, a simplified structure was constructed by replacing the methyl groups by $\mathrm{H}$ atoms in the crystallographic data. The complete active space (CAS) includes the unpaired electrons on the $\mathrm{Cu}$ and imvdz partners and the corresponding molecular orbitals (MOs), resulting in a minimal CAS[2,2] description. All CASSCF calculations were performed using the MOLCAS 7.0 package and available relativistic atomic natural orbital basis sets-type functions. ${ }^{29}$ The $\mathrm{Cu}$ and first coordination sphere atoms were described with $(21 \mathrm{~s} 15 \mathrm{p} 10 \mathrm{~d} 6 \mathrm{f}) /[5 \mathrm{~s} 4 \mathrm{p} 2 \mathrm{~d} 1 \mathrm{f}]$ and $(14 \mathrm{~s} 9 \mathrm{p} 4 \mathrm{~d}) /[3 \mathrm{~s} 2 \mathrm{p} 1 \mathrm{~d}]$ basis set contractions, respectively. A similar extended basis set $(14 \mathrm{~s} 9 \mathrm{p} 4 \mathrm{~d}) /[3 \mathrm{~s} 2 \mathrm{p} 1 \mathrm{~d}]$ was used for all the imvdz atoms, whereas a smaller [3s2p] basis set was chosen for the carbon atoms of the hfac ligands. Finally, the $\mathrm{H}$ atoms were depicted with a minimal basis set $(8 \mathrm{~s}) /[1 \mathrm{~s}]$. As previously mentioned, the $\mathrm{CF}_{3}$ groups of the hfac ligands can be replaced by $\mathrm{H}$ atoms. ${ }^{14}$ The dynamical polarization and correlation effects were then incorporated using the DDCI method as implemented in the CASDI code. ${ }^{30}$ Thus, selected configurations reached by excitations on top of the CASCI wave function were included following a second-order perturbation analysis as documented in literature. ${ }^{31}$ The DDCI philosophy relies on the simultaneous characterization of different states using a unique set of MOs, disposing of the nondifferential contributions involving the inactive-to-virtual double excitations. As previously reported, the singlet-triplet energy difference is almost insensitive to the $\mathrm{MO}$ definition and the triplet set of MOs was used. ${ }^{25,32,33}$ Three main CI spaces have been traditionally used in literature, referred to as DDCI-1, DDCI-2, and DDCI-3. The determinants in the DDCI space can be classified according to the number of inactive (holes, $h$ ) and virtual MOs (particles, $p$ ) implied in the excitation processes on the CAS determinants. In order to reduce the cost of computational time, dedicated MOs were constructed. From the DDCI-1 level of calculation, the difference between the singlet and triplet density matrices was diagonalized. This procedure allows one to identify the most contributing MOs to the singlet-triplet energy difference and to 


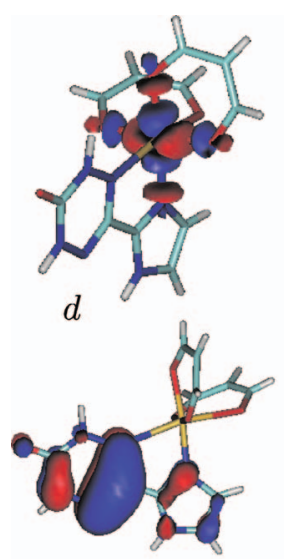

$\pi_{1}$

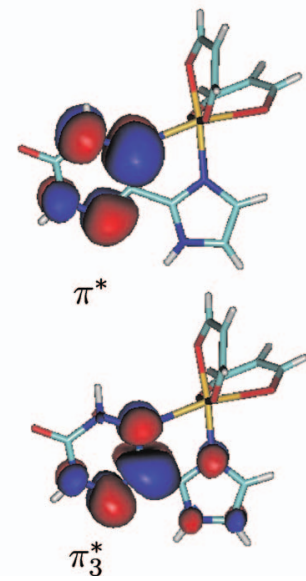

FIG. 2. Triplet CAS[2,2]SCF most relevant MOs orbitals. $d$ and $\pi^{*}$ correspond to the magnetic MOs (i.e., active MOs). $\pi_{1}$ and $\pi_{3}^{*}$ MOs govern the SP effects (see text).

neglect the rest. A significant reduction in the CI space results while the amplitude of the magnetic coupling constant is almost not affected. ${ }^{25,34-37}$

In order to elucidate the mechanisms governing the exchange coupling in (1), two complementary tools have been used. First, LOs were built from the triplet CASSCF MOs following the procedure reported in literature. ${ }^{38-41}$ The LOs offer an intuitive Lewis-like description of the zeroth-order electronic distribution and then a valuable analytical tool. ${ }^{42}$ Here, they are used to selectively separate the $\sigma / \pi$ contributions of the two parts of the imvdz ligand. A second analytical strategy is to split the CI space into the different relevant classes of excitations. Together with the usual DDCI-n (n $=1-3$ ), it is possible to perform class-partitioned CI calculations by selecting among the seven generic types of excitations in the DDCI-3 space $(1 h, 1 p, 1 h 1 p, 2 h, 2 p, 2 h 1 p, 1 h 2 p)$ those that are included in the CI space. This allows for an identification of the relevant mechanisms and gives information about the nature and amplitude of the contributions to the $J$ value. $^{27}$

\section{RESULTS AND DISCUSSION}

\section{A. A calculation level-dependent magnetism}

Based on the crystal structure, we first performed CAS[2,2]SCF calculations for both $S=0$ and $S=1$ states. The expected magnetic orbitals mainly $\mathrm{Cu} 3 \mathrm{~d}\left(x^{2}-y^{2}\right.$ symmetry) and imvdz SOMO $\pi^{*}$ are shown in Fig. 2. One should note that the SOMO $\pi^{*}$ is purely localized on the vdz moiety. In contrast, the close in energy MOs $\pi_{1}$ (occupied) and $\pi_{3}^{*}$ (virtual) exhibit non-negligible contributions on the vdz substituent, namely, the imidazole group.

Due to the quasiorthogonality of the magnetic MOs, a ferromagnetic behavior can be anticipated. This is a bare valence-only description that offers a relatively good estimate $\left(9.3 \mathrm{~cm}^{-1}\right)$ of the experimental $J$ value, as seen in Table I. The CASCI wave function for the singlet state is essentially represented by the two neutral configurations $\left|\pi^{*} \overline{3 d}\right|$ and $\left|3 d \overline{\pi^{*}}\right|$, i.e., each active orbital bears one active electron, while ionic forms $\left|\pi^{*} \pi^{*}\right|$ and $|3 d \overline{3 d}|$ (charge transfers forms) have a negligible weight. Nevertheless, it is known that such description may not be well adapted since non-negligible contributions arising from instantaneous charge and spin redistribution (i.e., correlation effects) are likely to deeply modify the minimal active space picture. $^{14,25-27}$ Thus, subsequent DDCI-1, DDCI-2, and DDCI-3 calculations were performed using a single set of MOs as requested in the DDCI philosophy, namely, the DDCI-1 dedicated MOs. The singlet-triplet energy splitting evaluated at different levels of calculations is summarized in Table I. At this stage, let us mention that the DDCI-2 level does not bring any quantitative change in the energetics and structure of the wave functions. One puzzling observation is the change not only in the amplitude but also in the sign of the exchange coupling. Usually, DDCI-1 and DDCI-2 calculations improve the $J$ estimate with respect to the bare CASCI evaluation, recovering 50\%-60\% of the experimental value. ${ }^{25,26}$ However, no change in the nature of the interaction has been reported to date. The alternation ferromagnetic/antiferromagnetic/ferromagnetic as the level of DDCI calculations is improved clearly demonstrates the importance of DDCI-3. Besides, such trend evidently calls for a thorough analysis of the underlying mechanisms.

The CI spaces are constructed from selected excitations on top of the reference CAS wave functions. As mentioned above, the DDCI-1 space includes all the single excitations that afford for two main effects: the SP and the stabilization of the ionic forms. The former contribution was originally introduced in the analysis of magnetic properties of radical assemblies. ${ }^{43}$ It involves a triplet excitation $h \rightarrow p$ in the inactive part as discussed more recently. ${ }^{25}$ While its sign cannot be predicted a priori, the stabilization of the ionic forms produces always an antiferromagnetic contribution to $J$, allowing the pairing of the two electrons in the same MO. ${ }^{25-27}$ Since the singlet wave function in complex (1) is essentially represented by the neutral forms at all levels of calculation (see Table I), it is expected that the role of the single excita-

TABLE I. Calculated $J$ values $\left(\mathrm{cm}^{-1}\right)$ in (1) using different levels of calculations. Normalized projections of the singlet wave function on the CAS.

\begin{tabular}{lrllll}
\hline \hline Level & \multicolumn{1}{c}{$\begin{array}{c}\text { J } \\
\left(\mathrm{cm}^{-1}\right)\end{array}$} & $\left|\pi^{*} \overline{3 d}\right|$ & $\left|3 d \overline{\pi^{*}}\right|$ & $\left|\pi^{*} \overline{\pi^{*}}\right|$ & $|3 \overline{3 d}|$ \\
\hline CASCI & 9.3 & 0.7060 & 0.7060 & 0.0394 & -0.0377 \\
DDCI-1 & -12.5 & 0.7061 & 0.7061 & 0.0386 & -0.0354 \\
DDCI-3 & 6.3 & 0.7061 & 0.7061 & 0.0403 & -0.0359 \\
\hline \hline
\end{tabular}


TABLE II. $J$ values $\left(\mathrm{cm}^{-1}\right)$ and weights of the leading excitations in the CI space (outside the CAS) using the class-partitioned CI procedure. The $\Delta J$ values correspond to the difference with respect to (a) the CAS, (b) the DDCI-1 space, or (c) the DDCI-2 space.

\begin{tabular}{lccccc}
\hline \hline Level & $\begin{array}{c}J \\
\left(\mathrm{~cm}^{-1}\right)\end{array}$ & $\begin{array}{c}\Delta J \\
\left(\mathrm{~cm}^{-1}\right)\end{array}$ & $\sigma \rightarrow 3 \mathrm{~d}$ & $\pi_{1} \rightarrow \pi^{*}$ & $\pi_{1} \rightarrow \pi_{3}^{*}$ \\
\hline CASCI & 9.3 & $\ldots$ & $\ldots$ & $\ldots$ & $\ldots$ \\
CAS+1 $h$ & 8.1 & $-1.2(\mathrm{a})$ & $<0.001$ & $<0.001$ & $\ldots$ \\
CAS+1 $p$ & 9.1 & $-0.2(\mathrm{a})$ & $\ldots$ & $\ldots$ & $\ldots$ \\
CAS+ $(1 h 1 p)$ & -9.1 & $-18.4(\mathrm{a})$ & $\ldots$ & $\ldots$ & 0.1117 \\
CAS+SP & -16.2 & $-25.5(\mathrm{a})$ & $\ldots$ & $\ldots$ & 0.1399 \\
DDCI-1 & -12.5 & $-21.8(\mathrm{a})$ & 0.0102 & 0.1051 & 0.1481 \\
DDCI-2 & -13.1 & $-0.6(\mathrm{~b})$ & 0.0104 & 0.1051 & 0.1481 \\
DDCI-2+(1h2p) & -2.3 & $10.8(\mathrm{c})$ & 0.0090 & 0.0409 & 0.0797 \\
DDCI-2+(2h1p) & 4.4 & $17.5(\mathrm{c})$ & 0.1150 & 0.1006 & 0.0842 \\
CAS+ $(2 h 1 p)$ & 10.5 & $1.2(\mathrm{a})$ & $\ldots$ & $\ldots$ & $\ldots$ \\
CAS+ $1 h+(2 h 1 p)$ & 17.3 & $8.0(\mathrm{a})$ & 0.1104 & 0.0590 & $\ldots$ \\
DDCI-3 & 6.3 & $19.4(\mathrm{c})$ & 0.0803 & 0.0550 & 0.0495 \\
\hline \hline
\end{tabular}

tions will be mainly governed by the SP effects. This is precisely what we will illustrate in Sec. III B.

Even though (1) exhibits antiferromagnetism at a DDCI-1 level, a ferromagnetic behavior is recovered at the best level (DDCI-3 in Table I). This observation is to be contrasted with previous theoretical inspections performed upon the $\mathrm{Ni}(\mathrm{II})$ and $\mathrm{Mn}(\mathrm{II})$ analogs. ${ }^{14}$ In particular, the ferromagnetic behavior in the $\mathrm{Ni}(\mathrm{II})$ compound is reproduced at a DDCI-1 level. Despite their demanding character due to the size and low symmetry of the system, the DDCI-3 calculations are in very good agreement with the experimental exchange coupling constant value. Thus, we believed that these observations deserved particular attention to unravel (i) the magnetically relevant parts and (ii) the leading mechanisms in complex (1).

\section{B. Detailed analysis of the exchange mechanism}

Let us first concentrate on a splitting of the wave function that can be obtained by considering separately the different types of excitations on top of the CASCI wave function. This particular analysis has been recently reported in literature $^{27}$ and allows us to specify the nature of the contributions (ferromagnetic versus antiferromagnetic) with respect to the classes of excitations. It is important to notice that this tool is just a guideline of the factors governing the coupling since higher-order effects resulting from the interactions of determinants belonging to different classes are also taken into account in the DDCI-3 space. These contributions are, in general, nonadditive since a variational treatment is performed within each subspace. With this remark in mind, the results following such strategy are summarized in Table II.

The DDCI-1 and DDCI-2 calculations give similar behavior, which indicates that the main effect is already present in the DDCI-1 space. Among the set of single excitations included in this space $(1 h, 1 p$, and $1 h 1 p)$, only the $1 h 1 p$ determinants bring a strong antiferromagnetic contribution $\left(-18.4 \mathrm{~cm}^{-1}\right)$ to the exchange coupling constant [CAS $+(1 h 1 p)$ entry in Table II]. Among the $1 h 1 p$ determinants, those taking into account the differential effect of the SP are responsible for the antiferromagnetic $J$ value obtained at a DDCI-1 level (CAS +SP in Table II). Let us mention that a rather different scenario was observed in the $\mathrm{Ni}$ (II) analog where the SP brings a leading ferromagnetic contribution. In fact, a closer inspection of the wave functions shows that the leading excitation on the DDCI-1 space corresponds to the $\left|\pi_{1} \pi_{3}^{*} \pi^{*} 3 d\right|$ determinant, which belongs to this CAS + SP subset $(0.1481$ weight in the singlet wave function, DDCI-1 entry in Table II). Interestingly, both $\pi_{1}$ and $\pi_{3}^{*}$ orbitals are mostly localized on the verdazyl part of the imvdz radical (see Fig. 2). Nevertheless, contributions upon the imidazole substituent are also present, in contrast with the SOMO nature.

Since the types of excitations governing the antiferromagnetic contribution have been isolated, let us now analyze which one recovers the ferromagnetic nature of the interaction inside the DDCI-3 space. This space includes two extra sets of excitations, named $2 h 1 p$ and $1 h 2 p$, which differ in the number of electrons in the active space. Using the classpartitioned CI procedure, it is possible to separate their individual effects on $J$. While both sets of determinants introduce a ferromagnetic contribution to the exchange coupling constant as compared to the DDCI-2 $J$ value, the largest one comes from the $2 h 1 p$ determinants [DDCI- $2+(2 h 1 p)$ entry in Table II]. This effect is not related to the direct interaction of the $2 h 1 p$ excitations with the valence-bond forms. In fact, they only produce a $1.2 \mathrm{~cm}^{-1}$ ferromagnetic contribution when coupled to the CAS determinants $[\mathrm{CAS}+(2 h 1 p)$ entry in Table II]. Their ferromagnetic contribution is associated to more complex pathways involving simultaneously other types of excitations. Additional evidence is obtained from the inspection of the wave functions. The dominant excitation on the DDCI- $+(2 h 1 p)$ and DDCI-3 wave functions is the LMCT $1 h$-type imidazole $\rightarrow$ Cu excitation $(\sigma \rightarrow 3 d$ column in Table II). While its contribution is rather negligible at a DDCI-1 level, the presence of the $2 h 1 p$ determinants produces an enhancement of this LMCT form. Among the wide set of $2 h 1 p$ determinants, the most participating ones correspond to single excitations on top of this LMCT forms, which precisely take into account its relaxation (i.e., its sta- 
bilization). It is known that LMCT forms are of lower energy for the triplet than for the singlet state. ${ }^{27}$ This could directly suggest that the coupling between $2 h 1 p$ and LMCT forms favors the triplet state, and consequently gives rise to a ferromagnetic contribution. However, this picture is valid only in the absence of any other competing antiferromagnetic pathways. The leading ones involve the $1 h, 2 h 1 p$, and the ionic forms. They become dominant when the ionic configurations have non-negligible weights in the singlet wave function. For ferromagnetic systems such as (1) where the singlet wave function is essentially represented by the neutral forms (see Table I), these mechanisms do not play any role, but the $2 h 1 p$ determinants can bring in ferromagnetic contributions. Finally, their roles can be isolated by means of CI calculations on a selected CAS $+1 h+(2 h 1 p)$ space. As shown in Table II, this space produces an enhancement of the ferromagnetic coupling as compared to the CAS $+(2 h 1 p)$, supporting the role of the underlined mechanism. In Sec. III C, we will provide additional proofs of the stability of the triplet LMCT forms and their role on the ferromagnetic pathways.

Therefore, the magnetic behavior of (1) can be seen as a balance of two main ferromagnetic contributions coming from (i) the orthogonality of the magnetic orbitals $\left(\sim 9 \mathrm{~cm}^{-1}\right)$, (ii) the $2 h 1 p$ excitations $\left(\sim 17 \mathrm{~cm}^{-1}\right)$, and (iii) one main antiferromagnetic contribution coming from the $1 h 1 p$ excitations $\left(\sim-18 \mathrm{~cm}^{-1}\right)$. The fortuitous cancellation of the two latter phenomena explains why the CASCI level leads to a correct evaluation of the exchange coupling in this particular system. As previously reported, ${ }^{25,26}$ a DDCI-3 level of calculations is required on top of the CASCI results to reach spectroscopic accuracy. However, our inspection clearly demonstrates that the understanding of the nature of the interaction significantly benefits from the here-conveyed analysis. To complement the wave function analysis, we carried out calculations, taking advantage of LOs, in order to identify the magnetically relevant parts of the imvdz radical.

\section{No one is innocent!}

From the previous wave function inspection, it appears that some classes of mechanisms are likely to bring some important ferromagnetic contributions. Nevertheless, one may wonder how magnetically relevant are the different moieties of the complex. The possible entanglement due to the presence of different chemical groups can be analyzed to provide a guide for future experimental works. Therefore, we then looked into the participation of the different parts of the imvdz radical ligand in the magnetic interaction. With this goal in mind, different fragments of the radical were frozen in the construction of the CI space and the resulting singlettriplet energy differences were compared to the reference DDCI-3 $J$ value given in Table II. Our results are summarized in Table III. First, our analysis confirms that the main antiferromagnetic contribution is provided by the SP mechanism on the verdazyl moiety. Let us concentrate on the $1 h 1 p$ excitations that account for a $\Delta J=-18.4 \mathrm{~cm}^{-1}$ antiferromagnetic contribution with respect to the CAS singlet-triplet splitting (see Table II). This contribution is reduced to $\Delta J=-11.8 \mathrm{~cm}^{-1}$ (see Table III) when the $\pi_{1} \rightarrow \pi_{3}^{*}$ excitation
TABLE III. Calculated $J$ values $\left(\mathrm{cm}^{-1}\right)$ in (1) by selectively freezing different parts of the imvdz ligand.

\begin{tabular}{lccrr}
\hline \hline \multicolumn{2}{c}{ Frozen orbitals } & CAS $+(1 h 1 p)$ & DDCI-1 & DDCI-3 \\
\hline vdz part & Imidazole part & & & \\
$\pi_{1}$ and $\pi_{3}^{*}$ & & -0.8 & -2.5 & 10.1 \\
& All $(\sigma$ and $\pi)$ & $\ldots$ & 0.2 & 7.1 \\
$\pi$ system & $\pi$ system & $\ldots$ & 1.9 & 10.6 \\
\hline \hline
\end{tabular}

is frozen (see Fig. 2). Since the $\pi_{1}$ and $\pi_{3}^{*}$ MOs are mostly localized on the verdazyl moiety, the antiferromagnetic contribution can be mainly attributed to the $\pi$-verdazyl network. Then, as soon as the imidazole LOs are frozen, the comparison between the DDCI-1 values given in Tables I and III is very instructive. A much greater variation in $J$ from DDCI-1 to DDCI-3 (18.8 in Table I versus $6.9 \mathrm{~cm}^{-1}$ in Table III) is evaluated when the charge density fluctuations upon imidazole are turned on. Thus, part of the understanding may be found in a picture attributing a bridging role to the imidazole part between the vdz and $\mathrm{Cu}$ ion spin-holding partners. In this respect, $a b$ initio $\mathrm{CI}$ calculations are very valuable since the wave function gives access to the amplitudes of such mechanisms. As previously observed, a significant $\sigma$-LMCT from the imidazole to the $\mathrm{Cu}$ center grows and formally results in a diradical species [im $\left.{ }^{\circ} \mathrm{vdz}^{\circ}\right]^{+}$and $\mathrm{a} \mathrm{Cu}^{+}$ion. This mechanism brings a strong ferromagnetic contribution since the imvdz SOMO is $\pi$-character, i.e., orthogonal to the mentioned $\sigma$-type LMCT. To support this point, complementary DDCI-3 calculations were performed upon the cation diradical $\left[\mathrm{im}{ }^{\circ} \mathrm{vdz}^{\circ}\right]^{+}$species in the presence of a closed-shell $\mathrm{Cu}^{+}$ ion. As summarized in Fig. 3, the ground state of the LMCT $\left.\mathrm{Cu}^{+}\left(\mathrm{im}^{\bullet} \mathrm{vdz}\right)^{+}\right)^{+}$is triplet and the first excited singlet state lies $850 \mathrm{~cm}^{-1}$ higher in energy at a DDCI-3 level. The mixing of the dominant $\mathrm{Cu}^{2+}$ (imvdz) electronic configuration with the $\mathrm{Cu}^{+}\left(\mathrm{im}^{\circ} \mathrm{vdz}\right)^{+}$charge transfer configuration should favor a triplet ground state. However, this effect is not observed at DDCI-1 level due to (i) the competing antiferromagnetic contribution of the SP forms and (ii) the high energy of this LMCT form. Once this $\sigma$-LMCT form is stabilized (by the mixing with the $2 h 1 p$ at DDCI-3 level), its weight is enhanced and leads to a greater stabilization of the triplet state

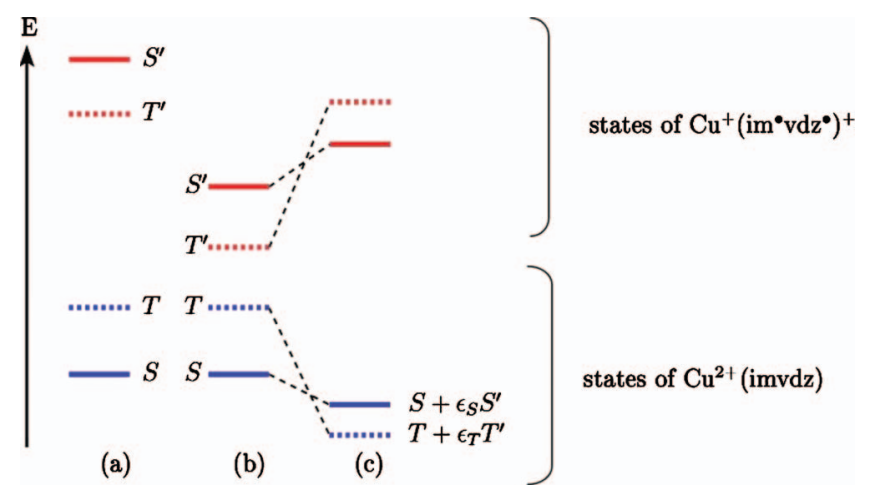

FIG. 3. Schematic spectroscopy of (1). (a) Diradical charge transfer $\mathrm{Cu}^{+}\left(\mathrm{im}^{\circ} \mathrm{vdz}^{*}\right)^{+}$spin states at a DDCI-1 level of calculation; (b) stabilization of the diradical spin states at a DDCI-3 level; (c) mixing of the diradical spin states into the $\mathrm{Cu}^{2+}(\mathrm{imvdz})$ states resulting in a triplet ground state for (1). 
of (1). A net ferromagnetic behavior results and can be seen as a gating of the weak $\pi$ kinetic exchange mechanism through a $\sigma$-LMCT mechanism. One of the key factor is the $\sigma / \pi$ orthogonality that simultaneously affords a low-lying triplet state on the diradical (Hund's rule) and suppresses the expected superexchange mechanism. Our analysis upon this verdazyl-based ligand highlights the bridging role of the imidazole moiety between the $\mathrm{Cu}$ ion and vdz part.

Finally, let us mention that such decomposition was absent in our previous interpretation upon a series of verdazylbased $\mathrm{Mn}(\mathrm{II})$ and $\mathrm{Ni}(\mathrm{II})$ complexes. ${ }^{14}$ Despite the strong similarities, the intensities of the exchange interactions in the $\operatorname{Mn}\left(J=-63 \mathrm{~cm}^{-1}\right)$ and $\mathrm{Ni}\left(J=220 \mathrm{~cm}^{-1}\right)$ analogs are much larger and the leading contributions are brought in at a DDCI-1 level.

\section{CONCLUDING REMARKS}

Wave function-based DDCI calculations were performed to unravel the origin of the ferromagnetic behavior of the $\left[\mathrm{Cu}(\mathrm{hfac})_{2}(\mathrm{imvdz})\right]$ complex. Even though a qualitative crystal field description allows one to recover the experimental results, one can conclude that this naive inspection model leads to "lucky" ferromagnetic conclusion. As matter of fact, a more elaborate calculation is likely to change the nature of the interaction, a rather disappointing scenario. However, excellent agreement with experiment is reached in terms of nature and amplitude as soon as a DDCI-3 level is considered. Thanks to large CI calculations, we demonstrate that the quasiorthogonality of the magnetic orbitals is not sufficient to fully understand the interaction between the $\mathrm{Cu}$ (II) ion and imvdz ligand. The weak observed ferromagnetism is the result of a balance between three main contributions: the orthogonality of the magnetic orbitals (ferromagnetic), the SP of the verdazyl part (antiferromagnetic), and third order mechanism involving the verdazyl substituent (ferromagnetic). This study highlights the versatile behavior of the imvdz radical that acts simultaneously as a ligand, a magnetic partner, and a bridge between the two spin holders. The nonintuitive, noninnocence of the imidazole part suggests that modifications of this moiety may deeply change the magnetic behavior. Thus, our work aims not only at understanding the origin of ferromagnetic properties but also at providing guides for future experimental works.

\section{ACKNOWLEDGMENTS}

The authors thank the ANR "fdp magnets" Project for financial support and the IDRIS national center for computational facility. C.J.C. is indebted to the Ministerio de Ciencia e Inovacion of Spain for financial support through Project Nos. CTQ 2008-06644-C02-02 and CTQ 2009-07767.

\footnotetext{
${ }^{1}$ G. Gatteschi and R. Sessoli, Angew. Chem., Int. Ed. 42, 268 (2003).

${ }^{2}$ M. Verdaguer, A. Bleuzen, V. Marvaud, J. Vaissermann, M. Seuleiman, C. Desplanches, A. Scuiller, C. Train, R. Garde, G. Gelly, C. Lomenech, I. Rosenman, P. Veillet, C. Cartier dit Moulin, and F. Villain, Coord. Chem. Rev. 190-192, 1023 (1999).

${ }^{3}$ R. Clément, S. Decurtins, M. Gruselle, and C. Train, Monatsch. Chem. 134, 117 (2003)
}

${ }^{4}$ D. Maspoch, D. Ruiz-Molina, and J. Veciana, Chem. Soc. Rev. 36, 770 (2007)

${ }^{5}$ K. Fegy, D. Luneau, T. Ohm, C. Paulsen, and P. Rey, Angew. Chem., Int. Ed. 37, 1270 (1998).

${ }^{6}$ O. Kahn, Molecular Magnetism (Wiley-VCH, New York, 1993).

${ }^{7}$ A. Sadoc, C. de Graaf, and R. Broer, Phys. Rev. B 75, 165116 (2007).

${ }^{8}$ S. Messaoudi, V. Robert, N. Guihéry, and D. Maynau, Inorg. Chem. 45, 3212 (2006).

${ }^{9}$ B. Le Guennic, S. Petit, G. Chastanet, G. Pilet, D. Luneau, N. Ben Amor, and V. Robert, Inorg. Chem. 47, 572 (2008).

${ }^{10}$ C. J. Calzado, J. F. Sanz, and J.-P. Malrieu, J. Chem. Phys. 117, 5158 (2003).

${ }^{11}$ E. Bordas, R. Caballol, C. de Graaf, and J.-P. Malrieu, Chem. Phys. 309, 259 (2005).

${ }^{12}$ R. Bastardis, C. de Graaf, and N. Guihery, Phys. Rev. B 77, 054426 (2008).

${ }^{13}$ D. Herebian, K. E. Wieghardt, and F. Neese, J. Am. Chem. Soc. 125, 10997 (2003)

${ }^{14}$ J.-B. Rota, L. Norel, C. Train, N. Ben Amor, D. Maynau, and V. Robert, J. Am. Chem. Soc. 130, 10380 (2008).

${ }^{15}$ R. G. Hicks, M. T. Lemaire, L. K. Thompson, and T. M. Barclay, J. Am. Chem. Soc. 122, 8077 (2000).

${ }^{16}$ J. Jornet, M. Deumal, J. Ribas-Arino, M. J. Beapark, M. A. Robb, R. G. Hicks, and J. Novoa, Chem.-Eur. J. 12, 3995 (2006).

${ }^{17}$ L. Norel, F. Pointillart, C. Train, L.-M. Chamoreau, K. Boubekeur, Y. Journaux, A. Brieger, and D. J. R. Brook, Inorg. Chem. 47, 2396 (2008).

${ }^{18}$ D. J. R. Brook, V. Lynch, B. Conklin, and M. A. Fox, J. Am. Chem. Soc. 119, 5155 (1997).

${ }^{19}$ F. Pointillart, C. Train, P. Herson, J. Marrot, and M. Verdaguer, New J. Chem. 31, 1001 (2007).

${ }^{20}$ D. Luneau and P. Rey, Coord. Chem. Rev. 249, 2591 (2005).

${ }^{21}$ C. Train, L. Norel, and M. Baumgarten, Coord. Chem. Rev. 253, 2342 (2009).

${ }^{22}$ J.-B. Rota, B. Le Guennic, and V. Robert, Inorg. Chem. 49, 1230 (2010).

${ }^{23}$ B. D. Koivisto and R. G. Hicks, Coord. Chem. Rev. 249, 2612 (2005).

${ }^{24}$ J. B. Gilroy, B. D. Koivisto, R. McDonald, M. J. Ferguson, and R. G. Hicks, J. Mater. Chem. 16, 2618 (2006)

${ }^{25}$ C. J. Calzado, J. Cabrero, J. P. Malrieu, and R. Caballol, J. Chem. Phys. 116, 2728 (2002)

${ }^{26}$ C. J. Calzado, J. Cabrero, J. P. Malrieu, and R. Caballol, J. Chem. Phys. 116, 3985 (2002).

${ }^{27}$ C. J. Calzado, C. Angeli, D. Taratiel, R. Caballol, and J. P. Malrieu, J. Chem. Phys. 131, 044327 (2009).

${ }^{28}$ J. Miralles, O. Castell, R. Caballol, and J.-P. Malrieu, Chem. Phys. 172, 33 (1993).

${ }^{29}$ G. Karlström, R. Lindh, P.-A. Malmqvist, B. O. Roos, U. Ryde, V. Veryazov, P.-O. Widmark, M. Cossi, B. Schimmelpfennig, P. Neogrady, and L. Seijo, Comput. Mater. Sci. 28, 222 (2003).

${ }^{30}$ N. Ben Amor and D. Maynau, Chem. Phys. Lett. 286, 211 (1998).

${ }^{31}$ P. de Loth, P. Cassoux, J.-P. Daudey, and J.-P. Malrieu, J. Am. Chem. Soc. 103, 4007 (1981)

${ }^{32}$ C. J. Calzado, S. Evangelisti, and D. Maynau, J. Phys. Chem. A 107, 7581 (2003)

${ }^{33}$ J. Cabrero, C. J. Calzado, D. Maynau, R. Caballol, and J.-P. Malrieu, J. Phys. Chem. A 106, 8146 (2002).

${ }^{34}$ J. Miralles, R. Caballol, and J.-P. Malrieu, Chem. Phys. 153, 25 (1991).

${ }^{35}$ O. Castell, R. Caballol, V. M. Garcia, and K. Handrick, Inorg. Chem. 35, 1609 (1996)

${ }^{36}$ C. J. Calzado, J. F. Sanz, O. Castell, and R. Caballol, J. Phys. Chem. 101, 1716 (1997)

${ }^{37}$ C. J. Calzado, J. P. Malrieu, J. Cabrero, and R. Caballol, J. Phys. Chem. A 104, 11636 (2000).

${ }^{38}$ B. Bories, D. Maynau, and M.-L. Bonnet, J. Comput. Chem. 28, 632 (2007).

${ }^{39}$ B. Le Guennic, N. Ben Amor, D. Maynau, and V. Robert, J. Chem. Theory Comput. 5, 1506 (2009).

${ }^{40}$ F. Banse, J.-J. Girerd, and V. Robert, Eur. J. Inorg. Chem. 2008, 4786.

${ }^{41}$ D. Maynau, S. Evangelisti, N. Guihery, C. Calzado, and J.-P. Malrieu, J. Chem. Phys. 116, 10060 (2002).

${ }^{42}$ M. Kepenekian, B. Le Guennic, K. Awaga, and V. Robert, Phys. Chem. Chem. Phys. 11, 6066 (2009).

${ }^{43}$ H. M. McConnell, J. Chem. Phys. 39, 1910 (1963). 